\title{
ACUTE SUBDURAL HAEMATOMA ANALYSIS OF CLINICAL PROFILE AND OUTCOME
}

\author{
Bijit Gogoi ${ }^{1}$ \\ ${ }^{1}$ Assistant Professor, Department of Surgery, Gauhati Medical College.
}

\begin{abstract}
\section{BACKGROUND}

Acute subdural haematoma is a common type of intracranial haematoma occurring from head injuries. It is frequently associated with cerebral contusion or laceration. It carries a high mortality and morbidity despite advanced care in the management of head injury. In the treatment of head injuries the subgroup of patients having acute subdural haematoma respond relatively poorly to the most vigorous treatment, both conservative and surgical which is most depressing. In our study our aim is to have an insight into this problem.
\end{abstract}

\section{METHODS}

A series of 77 patients of acute subdural haematoma with GCS from 3-15 were evaluated. All patients had acute SDH with or without associated parenchymal lesions on CT scan. Patients expiring within six hours of admission were not included in this study. Results: Age of the patients ranged from 1-56 years; 50.6\% patients were in their 3rd and 4th decades. While 59.74\% of patients with age $<40$ years survived, only $20.77 \%$ patients above 40 years survived and this difference was statistically significant ( $<<0.01$ ). Out of total 77 patients, 62 (80.51\%) survived. Number of patients operated for SDH were 5. Of them 4 survived and 1 died.

\section{CONCLUSION}

Adults in their 3rd and 4th decades of life are the commonest victims of acute SDH. Majority of the patients who had small SDH were treated conservatively and they responded to the treatment favourably. In our series of operations on acute SDH the time of surgery did not show any significant difference of outcome.

\section{KEYWORDS}

Head Injury, Subdural Haematoma, Midline Shift, Craniotomy, Glasgow Outcome Scale.

HOW TO CITE THIS ARTICLE: Gogoi B. "Acute Subdural Haematoma Analysis of Clinical Profile and Outcome." Journal of Evolution of Medical and Dental Sciences 2015; Vol. 4, Issue 105, December 31; Page: 17031-17033, DOI: 10.14260/jemds/2015/2577

\section{INTRODUCTION}

Acute subdural haematoma is a common type of intracranial haematoma occurring from head injuries. It is frequently associated with cerebral contusion or laceration. It carries a high mortality and morbidity despite advanced care in the management of head injury. In comatose patients of GCS less than 8 , the mortality rate is $50-80 \%$.

The outcome is significantly influenced by natural factors like age, other observations and factors like pupillary abnormality, initial coma scale, associated extracranial injury and raised intracranial pressure. Finding on CT scans like midline shift, associated lesions, presence of subarachnoid haemorrhage and basal cisterns compression are also related to the outcome.

Also interval between trauma and evacuation of haematoma and type of surgery are significant prognostic factors. Our present study is to analyse the clinical/radiological and management related factors in influencing the outcome of patients with acute subdural haematoma following head injury.

Financial or Other, Competing Interest: None.

Submission 01-12-2015, Peer Review 02-12-2015,

Acceptance 12-12-2015, Published 31-12-2015.

Corresponding Author:

Dr. Bijit Gogoi,

H.No-7,

Basisthapur Lane 4,

Wireless, Beltola,

Guwahati-781028.

E-mail: bgogoi123@yahoo.co.in

DOI:10.14260/jemds/2015/2577

\section{MATERIALS AND METHODS}

A series of 77 consecutive patients of acute subdural haematoma with a GCS from 3-15 admitted and treated at Gauhati Medical College and Hospital during the period from 1st June 2005 to 2nd August 2006, were evaluated.

All patients had acute subdural haematoma with or without associated parenchymal lesions (Contusion, haematoma, subarachnoid haemorrhage and infarct) on CT scan. Patients expiring within six hours of admission were not included in this study.

Clinical profile of patients were recorded which included age, sex, mode of injury, post resuscitation GCS, pupillary response, associated medical illness, hypotension and any other associated injury. All patients underwent an immediate CT scan following admission and CT findings (Site, degree of midline shift, associated parenchyma pathology, cisternal effacement, presence of subarachnoid haemorrhage, intraventricular haemorrhage, infarcts) were noted.

Patients were taken up for surgery depending on the mass lesion (SDH), mass effect and clinical status. Moribund patients, those with GCS 3 and thin subdural haematomas, no significant midline shift were operated. Craniotomy with evacuation of the subdural haematomas was done in those who were operated. Rest of the patients were treated conservatively.

The patients received intravenous mannitol for 3-5 days followed by furosemide according to their need. The patients also received intravenous anticonvulsants (Phenytoin sodium). Antibiotics were given according to need. All complications were treated accordingly. Patient's outcome was recorded at discharge and at one month following 
discharge (According to Glasgow outcome scale). The data obtained were carefully analysed and the clinical features, radiological findings and the management were then correlated with the outcome.

\section{Glasgow Outcome Scale (Score) is:}

1. Good recovery (Resumption of normal life despite minor deficits).

2. Moderate disability (Disabled but independent, can work in sheltered setting).

3. Severe disability (Conscious but disabled, dependent for daily support).

4. Persistent vegetative (Minimal responsiveness).

5. Death.

For statistical analysis, outcome under the Glasgow outcome scale was divided into favourable (Good recovery and moderate disability) and unfavourable (Severe disability, persistent vegetative state and death).

\section{RESULTS}

The study consisted of 77 consecutive patients of acute subdural haematoma: GCS from 3-15. Age of the patients ranged from 1-56 years; $50.6 \%$ patients were in their 3rd and 4th decades. While $59.74 \%$ with age less than 40 years survived, only $20.77 \%$ above 40 years survived and this difference was statistically significant $(\mathrm{p}<0.01)$.

Mean age of survivors was 31.14 years and 30.16 years in those who expired. There were 65 males out of which 52 survived and 12 females out of which 10 survived and this difference was statistically insignificant $(p>0.05)$. Mode of injury were road traffic accidents in $63.63 \%$ and fall from height in $18.18 \%$ patients and assault in $18.18 \%$ patients. 36 patients were conscious and 41 unconscious at the time of admission.

According to Glasgow coma scale poor motor response (M1-M3) were seen in $19.48 \%$ of patients. Fate of patients who had M1 motor response were 1 survived. Survival was better in those with good motor scores. M4-M5 score patients were 25 in number and 76\% survived. M2-M3 score patients were 14 in number and only $42.85 \%$ survived and this difference is statistically significant $(\mathrm{p}<0.01)$.

Overall, 62 out of 77 patients (80.51\%) survived. Statistically speaking mean motor response in patients who survived were 4.74 and in those who expired were 3.03 . Pupillary abnormality correlated well with the outcome. Out of 69 patients with bilaterally reacting pupils $58(84.05 \%)$ survived.

Major associated injuries were seen in 13(16.88\%) patients out of 77 patients. 8 patients had associated long bone fractures which were confined mostly to fractures like fracture femur, fracture tibia and fibula, fracture radius or ulna and fracture humerus; 1 had associated electrical burn of forearm. 2 had fracture of cervical vertebra and 1 had fracture of lumbar vertebra.1 had associated amputation of foot. No patient had chest injury, abdominal visceral injury or fracture pelvis. Of the 13 patients with major associated injuries 9 patients (69.23\%) survived, while 4 patients $(30.77 \%)$ died. Of the 64 patients without major associated injuries, 54 patients (84.37\%) survived while 10 patients (15.63\%) died.

The survival of patients with major associated injuries with the survival of patients without major associated injuries is statistically insignificant $(p>0.05)$. Subdural Haematoma
(SDH) was seen on the right side in 36 patients and $80.55 \%$ of these survived and on the left side in 32 patients and $81.25 \%$ of these survived. Bilateral SDH was seen in 7 patients and $71.42 \%$ survived. Tentorial SDH was seen in 2 patients and $100 \%$ survived.

Midline shift was found in 14 patients. Survival was found to be inversely related to midline shift. Survival was $100 \%$ in patients with $1-5 \mathrm{~mm}$ shift and $50 \%$ in patients with $6-10 \mathrm{~mm}$ shift and $25 \%$ in those with $11-15 \mathrm{~mm}$ shift and the difference was statistically insignificant. ( $p>0.05$ ).

Bilateral basal cistern effacement was seen in $64.93 \%$ of patients and of these only $90 \%$ survived while $92 \%$ of patients with unilateral or no cisternal effacement survived.

A total of 28 patients (36.36\%) had Subarachnoid Haemorrhage (SAH) and survival was $20(71.42 \%)$ of patients. (20 of 28 patients) with presence of SAH survived while 42 $(85.71 \%)$ of patients without subarachnoid haemorrhage survived and this was found to be statistically insignificant ( $p>0.05$ ). Associated contusion was present in 49 patients $(63.63 \%)$ and its presence or absence was statistically insignificant in the outcome of patients. Only 15 out of 23 patients with diffuse brain swelling survived. Most of the patients were managed conservatively.

Cerebral infarct was seen in 1 patient and he expired; 2 patients had pneumocephalus and 1 survived and 1 expired. 4 patients had Extradural Haematoma (EDH); 2 patients had EDH on same side, 1 patient had EDH on opposite side and 1 patient had EDH with bilateral SDH. They were managed conservatively. Of them 3 survived and 1 died. The complications seen in the management were septicaemia, acute renal failure, chest infection and CSF rhinorrhoea. Out of total 77 patients, 62 survived (80.51\%).Number of patients operated for SDH were 5 . Of them 4 survived and 1 died. At discharge, accordingly to Glasgow outcome scale, in 62 patients, 56 patients $(90.32 \%)$ had good recovery and 6 patients $(9.68 \%)$ had moderate disability. At one month follow-up in 62 patients, 58 patients (93.55\%) had good recovery and 4 patients (6.45\%) had moderate disability. In patients who survived, mean duration of hospital stay was 11 days (range 1-67 days). Overall outcome as last recorded in 77 patients, 62 patients ( $80.52 \%$ ) had favourable recovery and 15 patients $(19.48 \%)$ had unfavourable recovery on account of their deaths.

\section{DISCUSSION}

A strong correlation is seen between age and outcome in patients with acute subdural haematoma. Increasing age has a poorer outcome in adults, but opposite may be true for children. Survival in our patients was better in those below 40 years of age. (59.74\% of patients of age less than 40 years survived while only $20.77 \%$ of patients above 40 years survived) and this difference was found to be statistically significant. Howard, et al. (1989.1) showed 18\% mortality in young and $66 \%$ mortality in old patients and showed a good correlation between age and outcome. Acute SDH in young patients may be an epiphenomenon, while in elderly patients the poor outcome may be because of impaired regenerative capacity of the atrophic and aging brain.

Wilberger, et al. (1991.1) showed that the most common mechanism of injury was automobile accidents (53\%) followed by falls $(37 \%)$. In our study, majority of patients $(63.63 \%)$ had road traffic accidents. 
The outcome is related to GCS/motor score in several studies and outcome worsens as motor response progresses from normal to abnormal response including flaccidity.

Wilberger, et al. $\left(1991 .^{2}\right)$ reported $74 \%$ mortality in patients with GCS of 3-5 and 36\% mortality in patients with GCS of $6-8$. In our study $42.85 \%$ of patients with motor response M2-M3 and 76\% patients with M4-M5 motor response survived. Mean motor response in our patients who survived was 4.74 and in those who expired was 3.03 . The presence of abnormal pupillary finding in patients of acute head injury has predictive value.

Braakman, et al. (1980.3) reported 54\% mortality in patients with one reactive pupil compared with $90 \%$ mortality in patients with both pupil nonreactive. In our study $84.05 \%$ with bilaterally reacting pupils survived. Thickness of haematoma, associated unilateral cerebral oedema and associated parenchyma damage contribute to midline shift. Kotwica and Brezezinski (1993.4) showed 42\% favourable outcome and $39 \%$ mortality rates with less than $1.5 \mathrm{~cm}$ midline shift and $52 \%$ mortality when this shift was from 1.5 to $3 \mathrm{cms}$. In our series there was correlation between midline shift and outcome, i.e. survival of $100 \%, 50 \%, 25 \%$ in patients with midline shift of $1-5 \mathrm{~mm}, 6-10 \mathrm{~mm}$ and $11-15 \mathrm{~mm}$ respectively. In an autopsy study of head injury, Kristiansen and Tandon $\left(1960.5^{5}\right)$ saw that acute subdural haematoma is rarely an isolated lesion. The extensive associated contusion and laceration may be the dominant feature in the patients and are of greater importance to the outcome.

Incidence of brain contusions with acute SDH ranges from $7 \%$ to $82 \%$. Favourable outcome was $32-58 \%$ with isolated SDH and $12-32 \%$ with multiple contusions. Our study had $88.23 \%$ survival with single contusion, $78.12 \%$ survival with multiple contusion. Seelig, et al. (1981.6) did not report significant differences in outcome of patients with and without associated contusion. The very high mortality associated with surgery of acute SDH led many neurosurgeons to delay operating on such patients.

Seelig, et al. (1981.6) reported a dramatic reduction in mortality to $30 \%$ with early surgery (within 4 hrs of injury) as compared to $85 \%$ with delayed surgery. Stone et al. (1986.7) found no significant difference in outcome in early surgery (60\% mortality) and delayed surgery $(75 \%)$ and same results were observed in our series too. It is generally agreed that denial of surgery during the first $24 \mathrm{hrs}$ or delaying surgery in the hope of improving the outcome has no basis.

\section{CONCLUSION}

Adults in their 3rd and 4th decades of life are the commonest victims of SDH. There is a significant difference in mortality between patients below and above 40 years of age. Road traffic accident is the commonest cause of acute subdural haematoma.

Initial motor response and pupillary abnormality correlated well with the outcome, while presence or absence of associated injuries did not influence outcome significantly. Midline shift affected the outcome, but the difference was not significant.

Majority of the patients with acute SDH had associated focal (Contusion) or global (Subarachnoid haemorrhage) involvement of the brain or both of these. Presence or absence of SAH did not correlate well with the outcome. Presence or absence of contusion did not show any significant difference in the outcome of patients. Presence of infarct on initial CT scan was associated with a poor outcome. Majority of the patients who had small subdural haematoma were treated conservatively and they responded to the treatment favourably. In our series of operations on SDH, the time of surgery did not show any significant difference of outcome.

\section{REFERENCES}

1. Howard III MA, Gross AS, Dacey RJ, et al. (1989). Acute subdural haematomas: An age dependent clinical entity. J Neurosurg 71:858-863.

2. Wilberger JE, Harris M, Diamond DL (1991). Acute subdural hematoma morbidity, mortality and operative timing. J Neurosurg 74:212-218.

3. Braakman R, Glepke GJ, Habbema JDF. Systematic selection of prognostic features in patients with severe head injury. Neurosurgery 1980;6:362-370.

4. Kotwica Z, Brzezinski J (1993). Acute subdural haematoma in adults: An analysis of outcome in comatose patients. Acta neurochir (Wien) 121:95-99.

5. Kristiansen K, Tandon PN. Diagnosis and surgical treatment of severe craniocerebral injuries. J Oslo city Hosp (Suppl) 1960;10:107-213.

6. Seelig JM, Becker DP, Miller JD, et al. (1981). Traumatic acute subdural hematoma. Major mortality reduction in comatose patients treated within four hours. N Engl J Med 304:1511-1518.

7. Stone JL, Lowe RJ, Janasson 0, et al. (1986). Acute subdural hematoma: direct admission to a trauma centre yields improved results. J Trauma 26:445-450. 